\title{
Periodontal Soft Tissue Expanders - A Review.
}

\author{
G.Harini, DR.G.Kaarthikeyan \\ (Intern,Saveetha Dental College,Saveetha University,India) \\ (Reader,Saveetha Dental College,Saveetha University,India)
}

\begin{abstract}
Insufficient regeneration of missing bone and soft-tissue may present aesthetic or functional problems in patients indicative for dental implant surgery by using several techniques like bone augumentation ,bone substitutes and guided tissue regeneration.Soft tissue expansion is a technique used by plastic and restorative surgeons to cause the body to grow additional skin, bone or the other tissues.Extensive bone augumentation procedures are frequently carried out prior to implant surgery.Preaugmentation of soft tissue expansion with expanders leads to higher functional microvessel density in the tissue above the augmentation material and thus to more rapid osseointegration and inceases the probability of success in peri-implant bone augmentation.[1]Also Extensive soft tissue mobilization is required to achieve a tension -free wound closure at the grafted site and thus avoid wound dehiscence and loss of bone graft.[2]
\end{abstract}

Keywords: Complications of expansion,Osmotic hydrogel expanders,Tissue expansion,Tissue expanders.

\section{Introduction:}

Tissue expanders are used for the reconstruction of soft-tissue, this device consists of silicone elastomer inflatable expander with a remote silicone elastomer injection dome.The round,rectangular,elliptical, crescent shaped expanders are availaible with smooth elastomer shell.Use of rectangular expander provides the most effective surface area gained when compared to the round or crescent.The size of the expander must be large enough to provide required expansive forces necessary to achieve the desired tissue agumentation on the resorbed alveloar ridge. The expander are for temporary subcutaneous or submuscular implantation and is not intended for use beyond six months.

Pre -existing infection should be treated before implantation of the expander.It is the responsibility of the surgeon to advise prospective patients or their representatives ,prior to surgery of the possible complications associated with the use of this product.Potential advantage of the tissue expander over other techniques include relative simplicity of the surgical procedure, use of adjacent tissue for similar color ,texture,sensation,elimination of distant donor -site morbitity ,minimal incisional scarring and reduced operative time.

\section{MATERIALS:}

All the tissue expanders used wer a type of $352-3070-\mathrm{S}$ with the volume of $0.7 \mathrm{ml}$ in a silcone envelope.A cross head mini screw was used to fixate the tissue expanders to the bone through the extension of the silicone envelope.The expander volume was determined before and after implantation.[20]

\section{Tissue Expansion Prior To Augumentation Of Resorbed Edentulous Ridges:}

Patients with a severely resorbed mandible often suffer from problems with the lower denture.These problems include insufficient retention of lower denture, intolerence to loading by mucosa ,pain ,difficulties with mastication and speech,loss of soft tissue support and altered facial appearance.[3]

Primary wound closure is essential for sucessful regeneration of bone.Soft tissue dehiscene and subsequent exposure of bone grafts to the oral cavity are complications of ridge augmentation are the main causes for insufficent outcomes of reconstructive surgery.Poor quality and quantity of soft tisssue may lead to difficulties in achieving primary closure of the flap which leads to graft exposure.

Tissue expansion improves the quality and quantity of soft tissue and facilities primary wound closure ,with a result of reduced incidence of wound dehiscene and post-operative exposure of bone grafts.

Usually tissue expansion and maturation are usually completed afte 6-8 weeks. The expander is removed in the course of augmentation surgery.Incision and flap design are chosen as required for the intended method of bone augmentation.It is possible to cut directly into the expander.

Patients with a severely resorbed mandible often suffer from problems with the lower denture.These problems include insufficient retention of lower denture, intolerence to loading by mucosa ,pain ,difficulties with mastication and speech,loss of soft tissue support and altered facial appearance. 


\section{Piezosurgery And Screw Expanders:} dentists.[4]

The functional rehabitilation for severely resorbed mandible has been a challenge for oral surgeons and

Piezosurgeries provides a micrometric cuts for minimally invasive surgeries with maximum surgical precision and intra-operative tactile sensation.It protects any kind of soft tissue,nerves and blood vessels without injuring it while cutting the bone and reduces post-operative discomfort to the patients at the same time.

Some cases may report with adequate bone height for implant placement but have a narrow edentulous ridges,in that case piezosurgery technique is carried out,which allows the expansion of narrow,anatomically limiting,atrophic ridges creating space for immediate implant placement,and the implants where restored to a functional and esthetic outcome [5]

This may allow for a shortened treatment time and the elimination of donor-site morbidity.

\section{Self-Inflating Tissue Expanders:}

Self-inflating tissue expanders were developed in parallel to balloon-type devices.[11]Currently these osmotic hydrogel expanders which help to generate additional soft tissue are subperiosteally inserted prior to bone grafting which may contribute to overall improvement in bone augumentation process ,it also limits the complications related to the lack of tissue and requires only a short surgical time which creates benefit in cost ,time and less pain.Self-inflating hydrogel tissue expanders heralded a significant advance in the reconstructive techniques available for the surgical restoration of a wide variety of soft tissue defects [8]Unlike conventional expanders ,hydrogel expanders need not be filled from external source and have good mechanical properties and does not cause inflammatory reactions.[6]

The self inflating material is a hydrogel consisting of a modified copolymer of methylmethacrylate and $\mathrm{N}$ - vinyl-2-pyrrolidone, which takes up water by osmosis.[7]However, their impact was hindered by their limited expansion volumes and potential complications. The adoption of biocompatible osmotically active hydrogels was a major advance in the evolution of self-inflating devices.[11]

Self-inflating tissue expanders are made up of specially developed hydrogel that uses the osmotic pressure to gain the volume.They were considered to be the reliable product for creating space between soft tissue and bone and the overlying soft tissue remained in excellent shape and there was no difference between the soft tissue volume and bone volume between the tunnel and flap approach after 40 days without any inflammation.[9]

Before expansion ,osmed hydrogel expanders are hard ,small and are easy to handle.After implantation ,they absorb the body fliuds and grow consistently to their predefined form and size.The expanders are sheathed with perforated silicon shell for controlled slow influx of body fluid and slow continous swelling. The increasing volume of the expander stimulates growth of the soft tissue.Perioperative adminstration of antibiotics is considered mandatory.The tissue expander is placed under the tunnel flap and secured with the bone fixation screw through the flap.

\subsection{ADVANTAGES :}

General advantages of osmed tissue expanders are safe material made up of cross linked hydrogel copolymers based on methyl methacrylate and $\mathrm{N}$-vinyl pyrrolidone,low complication rate,high biocompatibility being non-toxic ,on-genotoxic and non-immuno reactive ,material pureness and safety which ensures reliable quality,low risk of infection,minimal trauma, short surgical time, controlled swelling without pressure peaks.A short surgical time reduces the post-operative pain and saves the costs.Submucosal implantation of self -filling osmotic tissue expanders results in only momemtary disturbance of microcirculation.[10]Hydrogel expansion of the periosteum is an applicable method to achieve surplus amount of soft tissue to cover bone grafts.More refinements may be required in minimizing complications in smoking patients.[15]

4.2 Indications: Tissue expansion is indicated in bone regeneration procedures and onlay grafting procedures.

4.3 Contraindications:It is not recommended for systemic disorders such as uncontolled diabetes, heavy smoking,Intravenous medication of bisphosphonates.Other local contraindications are untreated gingivitis ,periodontitis ,caries .Insufficient oral hygiene and previous radiaton therapy.

\subsubsection{Types of osmed tissue expanders :cylinder and cupola.}

Osmed tissue expander cylinder dental are used for straight edentulous areas and osmed Tissue expander cupola dental are used for small one or two missing teeth region with curved frontal edentulous area. 


\subsubsection{Indications:}

Tissue expansion prior to extensive bone augmentation surgery e.g. onlay bone grafting with bone block grafts and other bone regeneration surgeries.

\subsection{Templates:}

Templates are used for selection of the appropriate tissue expander showing initial and final expander volumes.The templates facilitate the preparation of the recipient site during surgery.The cylindrical part of the template corresponds to the hydrogel core.The distance from the cylindrical tip to the bend corresponds to the length of the expander in its silicone shell.

\subsection{Phases in osmotic hydrogel soft tissue expansion:}

Phase I represents the biodegradable 'time switch' which delays the onset of swelling following implantation for a period of typically two weeks , as dictated by the surgeon.

The rate of osmotic expansion during Phase II is carefully controlled either by an integral polymer scaffold or an external semipermeable membrane thus preventing the undesirable effects of excessive expansion which include pain and potential tissue necrosis.

The final degree of swelling achieved at Phase III can be precisely controlled (up to $1500 \%$ if necessary) and is determined by the hydrogel formulation used. The device is entirely inert and will remain quiescent during Phase III until surgically removed in order to perform the definitive reconstruction.[11]

\subsection{Higher vascularisation with Tissue expanders:}

The use of osmed expanders prior to vrtical augmentation of resorbed edentulous ridges reduces the mean incidence of post-operative graft exposition from $25 \%$ to $4 \%$ and increases the bone gain from approximately $4.0 \mathrm{~mm}$ to $7.5 \mathrm{~mm}$ compared to augmentations without prior tissue expansion. The surrounding soft tissue is vascularized and tension-free closure is facilitated.[16]

4.7 Bone augmentation after soft-tissue expansion using hydrogel expanders:effects on microcirculation and oseointergration:

Soft tissue coverage can be achieved by without tension through the pre -augmentation tissue expansion with hydrogel expanders. It leads to higher microvessel density in the region above the augmented material and leads to more rapid oseointegration.[17]

\subsection{Submucosal implantation of soft tissue expanders does not affect microcirculation:}

Submucosal implantation of soft tissue expanders results in only momentary disturbances of microcirculation. The minor imparement of perfusion may explain the consitently good outcomes of submucosal implantation of these expanders.[18]

\subsection{Precautions:}

Patients should abstain from brushing the treated area,Surgical assistance must take care for a dry operative field in order reduce contamination of the tunnel and the expander.Damaged expanders must be discarded.Removable dentures should not be worn during tissue expansion,Temperory fixed partial dentures should be adjusted according the increasing volume regularly.

\section{Chitosan Soft Tissue Expanders:}

Chitosan ascorbate,obtained by mixing chitosan with ascorbic acid and sodium ascorbate,was produced in a gel form suitable for the treatment of periodontal problems according to current dental surgery.Chitosan ascorbate plays an important biological role in the presence of atmospheric oxygen and at $\mathrm{pH}$ of 6.Thus the proliferation and organization of the cells were favoured with the subsequent enhanced capability of recontsructing the soft tissue and reduces the occurenvce of tooth mobility and pocket depths.[19]

\subsection{Infection:}

\section{Inherent Surgical Risks Of Tissue Expander Surgery:}

Infection can occur within the expander pocket. Manifested with swelling and pain.In some cases, the infection responds to antibiotics, warm compresses, and partial implant deflation to minimize vascular compromise. If medical therapy fails to clear the infection, the implant should be removed, the pocket irrigated well, drained and allowed to heal.However,Prophylactic antibiotics may be considered for subsequent dental or other surgical procedures.In certain rare cases, with patients of weakend immunity toxic shock syndrome have been noted after tissue expander, which is a life threating condition. 
Periodontal Soft Tissue Expanders - A Review.

\subsection{Bleeding:}

Post-operative bleeding may occur and emergency treatment should be made to drain of the accumulated blood.Do not take aspirin or any anti-inflammatory medicatons as it may increase the surgical risk of bleeding

\subsection{Scarring:}

Although good wound healing after a surgical procedure is expected ,some amount of scarring is left after all surgical treatments.In some cases ,scars may require surgical revision or treatment.

\subsection{Allergic reactions:}

In some cases allergic reactions tend to occur due to the injected agents,topical preparations or the suture materials. Anaphylactic reactions may occur in response to the drugs given during surgery and postoperative medications.It requires an additional treatment.

\subsection{Pain:} duration.

Some of pain is experienced after tissue expander surgery which may be of a varying intensity and

\subsection{Hypoxia:}

Rapid expansion leads to high pressure peaks that may cause hypoxia in the tissue and that may lead to damage of the expanded tissue.[20]

\section{Conclusion:}

Patients who lack part or all jaw bone and soft tissue and are in need of a tooth implany, run at a greater risk of unsucessful operations. This is because there is not enough soft tissue to cover a bon transplat performed in anticipation of the tooth implant.The so called osmotic hydrogel expanders increases the amount of soft tissue , which increases the risk of likelihood of bone transplant.Advantages of the osmed tissue expanders are that the material is safe with low complication rate and low risk of infection due to small incision site and minimal trauma.[14]At present alloplastic material such as titanium is often used for mandibular reconstruction as it is resistant to corrosion, acceptable and biocompatible.[12]Controlled soft tissue expansion encourages the development of tissues which ideally match the color and texture to give a donor effect, which offers a new ,safe and widely applicable technique in reconstructive surgery.Synthetic implants or bone grafts have been sucessfully implanted beneath the expanded soft tissues.[13]

\section{References:}

[1]. Constantin Von See,Nils-Claudius Gellrich,Ulrike Jachmann,MatthiasW.Laschke,Kai-Hendrik Bormann,Martin Rucker,Bone augmentation after soft tissue expansion using hydrogel expanders:effects on microcirculation and osseointergration,Journal of Periodontol, Volume 21,Issue 8,pages 842-847, august 2010.

[2]. Dogan Kaner,Han Zhoa,Hendrik Terheyden,Wolfgang Arnold,Anton Friedmenn,Effect of soft tissue expansion on microcirulation and healing after vertical augmentation in dogs.grant 687-2010.

[3]. Stellingsma C,Vissink A,Meijer HJ,Kuier C,Raghoebar GM.Implantology and the severly resorbed edentulous mandible.Journal of Oral implantology,2004 jul 1;15(4):240-8.

[4]. Manfro R,Batassini F,Bortoluzzi MC.Severly resorbed mandible treated by soft tissue expansion(tent pole) grafts:case report.2008 Dec;17(4):408-13.

[5]. Andrew Kelly,DDS,Dennis Flanagaan,DDS.Ridge Expansion and Immediate Placement With Piezosurgery and Screw Expanders in Atrophic Maxillary Sites,Journal of Oral Implantology,Feburary 2013,vol.39,No.1,pp. 85-90.

[6]. Wiese KG,Heinemann DE,Ostermeier D,Peters JH.Biomaterial properties and biocompatibility in cell culture of a novel selfinflating hydrogel tissue expander.J.Biomed Mater Res 2001;54:179-88.

[7]. Wiese KG,Heinemann DE,Ostermeier D,Peters JH. Biomaterial properties and Biocompatibilty in cell culture of a novel selfinflating hydrogel tissue expander.J.Biomed Mater Res.2001 Feb;54(2):179-88.

[8]. Swan MC, Bucknall DG, Goodacre TE, Czernuszka JT.Synthesis and properties of a novel anisotropic self-inflating hydrogel tissue expander 2011 Mar;7(3):1126-32.

[9]. Uijlenbroek HJ,Liu Y,He JF,Visscher C,van Waas MA,Wismeyer D.Expanding soft tissue with osmed tissue expanders in the goat maxilla.Clin Oral Implants Res.2011 Jan;22(1):121-8.

[10]. Dogan Kaner,Han Zhao,Hendrik Terheyden,Anton Friedmann,Submucosal implantation of soft tissue expanders does not affect microcirculation.Clinical Oral Implants Research,18 April 2013 DOI:10.1111/clr.12158.

[11]. Soft Tissue Expansion Solutions for Reconstructive Surgery, Oxtex Intelligent Tissue Expander, 01 June 2012

[12]. Osmed Tissue Expander for dental surgery found. http://www.kanchanhealthcare.com/OTEProdInfo/OTE-Dental/osmed_Dentalsurgery_2010-01.pdf (5 October 2012, date last accessed.

[13]. Louis C.Argenta,MD,Controlled tissue expansion in reconstructive surgery, Michigan 48109,USA.

[14]. Singare S, et al. Design and fabrication of custom mandible titanium tray based on rapid prototyping. Med Eng Phy 2004;26:6716.

[15]. Peter Abrahamson,Dan-Ake Walivaara,Sten Isaksson,Gunilla Andersson.Periosteal expansion Before local Bone Rconstruction Using a New Technique For Measuring Soft tissue Profile Stability:A Clinical Study.Journal of Oral and Maxillofacial Surgery, Volume70,Issue 10,October 2012,Pages e521-e530. 
[16]. Dogan Kaner and Anton Friedmenn,Soft tissue expansion with the self-filling osmotic tissue expanders before vertical augmentation:a proof of principal study.Journal of Periodontology, 2 NOV 2010,DOI:10.1111/j.1600-051x.2010.01630.x.

[17]. Von see C,Gellrich NC,Bormann KH,Rahmann A,Rucker M,Int J Oral Maxillofac Implants 2010;25:979-984

[18]. Kaner D,Zhoa H,Terheyden H,Friedmenn A.Clin Oral implants, Res.2013APR 18 ,DOI 10.1111/clr.12158.

[19]. Riccardo Muzzarelli,Graziella Biagini,Armanda Puganaloni,Oscar Filippini,Venanzio Baldassarre,Reconstruction of parodontal tissue with chitson,Faculty of Medicine.

[20]. Henri J.J.Uijlenbrooek, Yuvelian Liu,Jian Feng He,Cornie Visscher,Marinus A.J.van Waas,Daniel Wismeyer,Expanding soft tissue with Osmed tissue expander in goat maxilla,Department of Oral Implantology and Prosthodontics.13 April 2010.doi:10.1111/j.1600-0501.2010.01972.x. 